\title{
Tunable Spreading and Shrinking on Photocontrolled Liquid Substrate
}

\author{
Wenjie Ji, ${ }^{\dagger}+$ Weibin Li, ${ }^{\dagger, \ddagger 0}$ Yuren Wang, ${ }^{\dagger,+}$ and Ding Lan ${ }^{*}, \dagger,+0$ \\ ${ }^{\dagger}$ Key Laboratory of Microgravity (National Microgravity Laboratory), Institute of Mechanics, Chinese Academy of Sciences, 100190 \\ Beijing, China \\ ${ }^{\ddagger}$ School of Engineering Science, University of Chinese Academy of Sciences, 100049 Beijing, China
}

Supporting Information

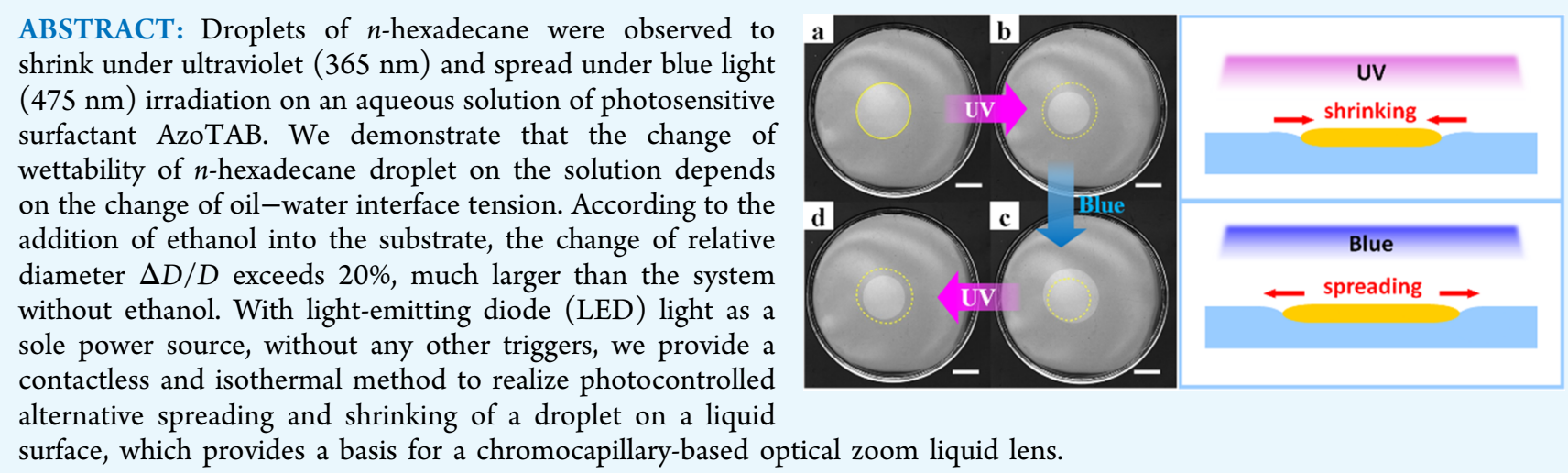

\section{INTRODUCTION}

The spreading coefficient is usually used for describing the mechanisms of one liquid spreading on the surface of another, which is defined as $S=\sigma_{\mathrm{w}}-\left(\sigma_{\mathrm{o}}+\sigma_{\mathrm{ow}}\right)\left(\sigma_{\mathrm{w}}, \sigma_{\mathrm{o}}\right.$, and $\sigma_{\mathrm{ow}}$ refer to the air-water surface tension, the air-oil surface tension, and the oil-water interface tension, respectively). It is a thermodynamics criterion to distinguish different types of wetting (for the case of short-range forces): a complete wetting regime where a drop spreads out into a molecular-thickness membrane with $S>0$ and a partial wetting regime where a drop spreads into a finite liquid lens with $S<0 .{ }^{1}$ If the spreading coefficient $S$ changes and keeps negative value $S<0$, the partial-wetting droplet would extend for $\Delta S>0$ and shrink for $\Delta S<0 .^{2}$ Therefore, the change of spreading coefficient $(\Delta S)$ in a system would lead to the change of wettability and the motion of contact line, and it can be realized by adjusting the relevant interface tension in this formula. Until now, there have been many methods to induce the change of interfacial tension, which are summarized into three categories including creating surface micro-/nanostructure, controlling chemical composition (or chemical components concentration), ${ }^{3-5}$ or using external field stimuli.

For solid substrates, the studies on special surface with micro-/nanostructures developed rapidly, especially natural interfaces including lotus leaves, water-strider legs, and the elytra of aquatic beetles, etc. ${ }^{6-8}$ Besides, various approaches for the preparation of fine surface geometrical structures were proposed, such as template synthesis, ${ }^{9}$ phase separation, ${ }^{10}$ electrochemical deposition, ${ }^{11}$ electrohydrodynamics, ${ }^{12}$ crystallization control, ${ }^{13}$ chemical vapor deposition, ${ }^{14}$ self-assembly, ${ }^{15}$ and so on. In addition, chemical component concentration gradients can be generated in many ways including asymmetric dissolution (for instance, in the "camphor boat" experiment), ${ }^{16}$ electrochemical control, ${ }^{17,18}$ interfacial chemical reaction, ${ }^{19} \mathrm{pH}$ gradient, ${ }^{20}$ etc. Undoubtedly, there are various methods to adjust interface tension but, nonetheless, these methods are usually irreversible. To realize the controllable wetting and tunable interface tension, the strategy of using external stimuli is developed. The most common external stimulus is based on thermal (thermocapillary effect), ${ }^{21-25}$ electrical (electrowetting effect), ${ }^{26-30}$ electrochemical effect, ${ }^{31}$ etc. The thermal-capillary effect is based on a thermal-induced change of interface tension. Temperature gradients were exploited to generate interfacial gradients which can induce interfacial liquid flow toward higher interfacial tension. The other well-known example is the electrowetting effect that the liquid-solid interfacial tension is electrostatically controlled. The contact angle (CA) and interface tension of the conducting liquid on a nonconductive surface are varied by applying a potential difference between the liquid and the surface.

In recent years, light irradiation is studied as an important external stimulus to switch surface wettability reversibly. Under ultraviolet (UV) irradiation, various light-responsive inorganic oxides and organic polymers could change from large contact angles (CAs) to relatively low CAs and return to their original state in darkness or under visible light. The first type of

Received: September 17, 2019

Accepted: November 25, 2019

Published: December 11, 2019 


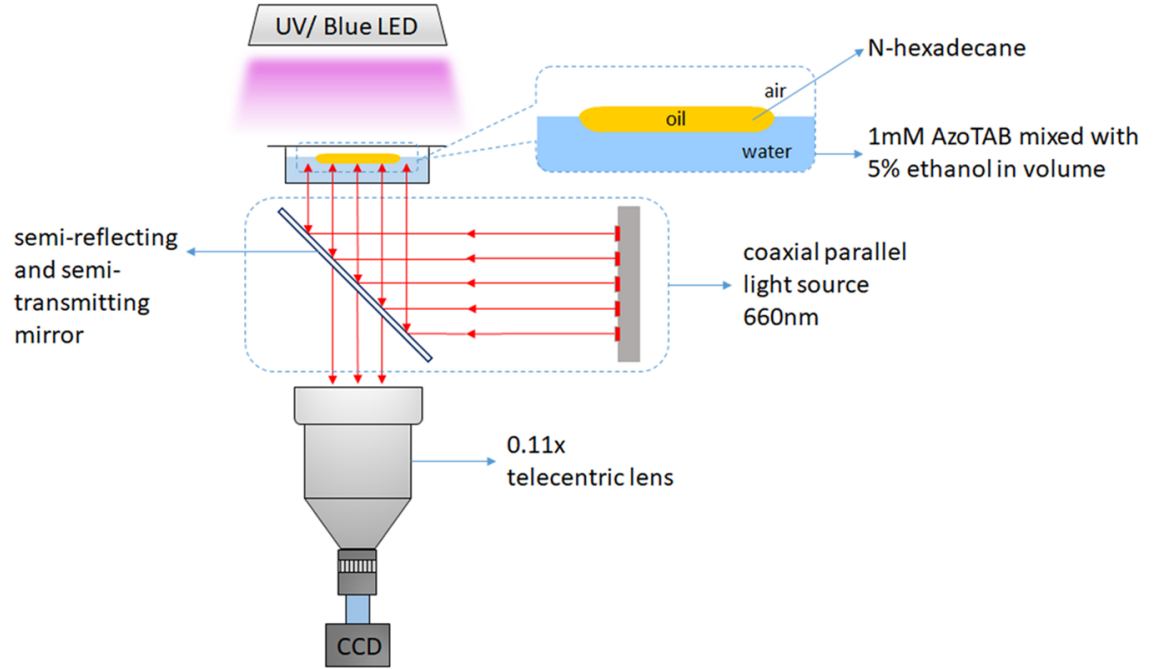

Figure 1. Sketch of the experimental setup used for the observation of droplet wetting. The solution could be irradiated by UV/blue light using the surface light source above the observation setup. The relevant data are listed as follows: surface tension of air-oil, $\sigma_{\mathrm{o}}=27.8 \mathrm{mN} / \mathrm{m} ;$ air - water, $\sigma_{\mathrm{w}}=$ $57.9 \mathrm{mN} / \mathrm{m}$; oil-water, $\sigma_{\mathrm{ow}}=33.9 \mathrm{mN} / \mathrm{m}$; and spreading coefficient $S=\sigma_{\mathrm{w}}-\left(\sigma_{o}+\sigma_{\mathrm{ow}}\right)=-2.8 \mathrm{mN} / \mathrm{m}$.

substrates is light-responsive inorganic oxides. The titanium dioxide $\left(\mathrm{TiO}_{2}\right)$ substrates were first found to have a photosensitive effect. Their photosensitive wettability was reported by Fujishima et al. in $1997,{ }^{32}$ and relevant research about wetting behaviors on titanium dioxide $\left(\mathrm{TiO}_{2}\right)$ were carried out over the next few years. ${ }^{33,34}$ Moreover, reversible wettability transition in complex environments, such as underwater or oil, draw people's attention. Switchable underwater superoleophobicity-superoleophilicity on lasermodified titanium surfaces was reported in $2015 .^{35}$ Recently, Yang et al. reported a fast optical induced wetting states transition surface achieved by atomic layer deposition of $\mathrm{ZnO}$ on micropillared glass substrate surfaces, enabling tens of seconds transitions for a wetting/dewetting cycle, shortened from an hour as typically reported. ${ }^{36}$ The other kind of photosensitive wettability on solid substrate is achieved by the organic compound layer with photochromic moiety. For instance, incorporation of azobenzene into a solid surface composition provides photodependent wettability. ${ }^{37-40}$ After that, azobenzene containing surfactants were developed rapidly, which played an important part in recent researches.

In comparison with the photosensitive solid substrate, it could be imaged that if a liquid contained photosensitive organic matter with photochromic functional groups, the interfacial characterization could be controlled by light illumination with different wavelengths. In terms of mechanism, chromocapillary effect is a light-induced Marangoni effect, which was first reported by Diguet in $2009 .^{41}$ In recent years, photosensitive surfactants modified with an azobenzene moiety have played the role of a transducer mediating between different states of size, shape, surface energy, and spatial arrangement of various nanoscale soft-material systems. ${ }^{42}$ AzoTAB is one kind of most commonly used photosensitive surfactant containing an azobenzene moiety. It is in a trans, apolar configuration under dark or blue light, while it isomerizes into cis, polar configuration under UV condition. Both isomers can be easily and quickly converted from trans to cis or cis to trans upon UV $(365 \mathrm{~nm})$ and blue light $(475 \mathrm{~nm})$, respectively. ${ }^{43}$ The trans isomer exhibits a higher affinity for the surface than the cis isomer, which is easier to desorb from the surface. ${ }^{44}$ Photosensitive surfactants are extensively applied in fields of photomanipulation of liquids, ${ }^{41,45-47}$ fundamental investigations in biology, ${ }^{48,49}$ physics, ${ }^{50-52}$ or chemistry. ${ }^{53-55}$ They provide a foundation to realize reversible photoisomerization of molecules by incorporating an azobenzene moiety into a molecule. These two different molecule polarities would lead to two different molecule behaviors. Therefore, this kind of photosensitive surfactant molecules offers a new possibility that a macroscopic one-way physical process might be tuned and reversed by light irradiation with different wavelengths.

In this article, we are going to introduce a noncontacting method to adjusting interface tension in an isothermal and wavelength-dependent manner based on "chromocapillary effect". It is driven by surface tension gradient similar to the thermocapillary effect, but the only difference is that the presence of the photosensitive surfactant rather than temperature drives it. We find that $\mathrm{UV} /$ blue light can be used to adjust the spreading coefficient $S$ to control wetting behavior of a microliter-sized $n$-hexadecane droplet on the surface of AzoTAB solution. We observed alternative shrinking and spreading of $n$-hexadecane droplet under UV and blue light, respectively. It is attributed to different interfacial properties under UV and blue light, which depend on the different adsorption properties of two isomers (trans or cis). Based on the principle of thermodynamics, we use the spreading coefficient to explain this phenomenon preliminarily, which may provide a potential method for a chromocapillary-based optical zoom liquid lens.

\section{EXPERIMENTAL SECTION}

2.1. Materials. $N$-hexadecane (purity AR $98 \%, \rho=0.77 \mathrm{~g} /$ $\mathrm{cm}^{3}$, viscosity $\mu=3.451 \mathrm{mpa} \cdot \mathrm{s}$, surface tension $\sigma=26.8 \mathrm{mN} / \mathrm{m}$ at $20{ }^{\circ} \mathrm{C}$ ) was purchased from Aladdin Industrial Corporation in Shanghai, China. The deionized water was produced by a MilliQ Academic filtering system, Millipore Inc. To obtain a light-sensitive surface, a cationic photosensitive azobenzene trimethylammonium bromide surfactant (AzoTAB) was dissolved in the aqueous substrate.

2.2. Experimental Methods. $N$-hexadecane is chosen as a floating droplet owing to its nonvolatility, chemical stability, insolubility in water, especially its negative spreading 


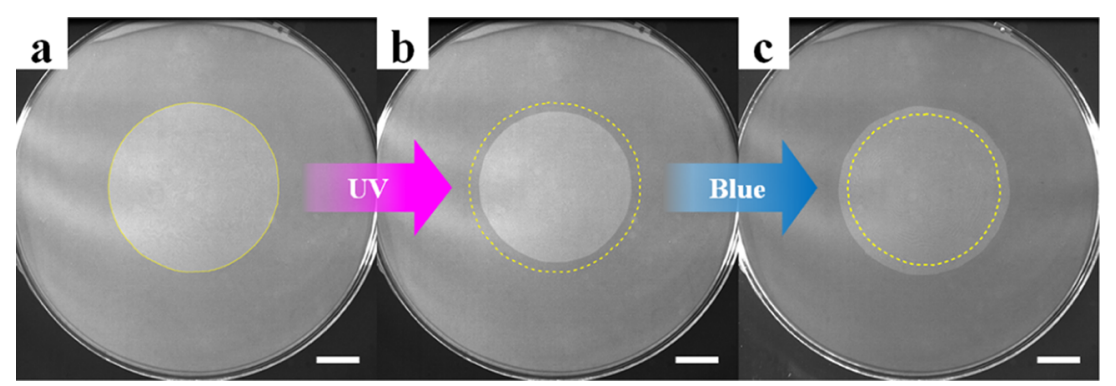

Figure 2. Three successive states of $10 \mu \mathrm{L}$ of $n$-hexadecane on $1 \mathrm{mM}$ AzoTAB solution is shown: (a) initial state; (b) the state after $120 \mathrm{~s}$ UV irradiation; and (c) the state after $180 \mathrm{~s}$ blue light irradiation. The solid line is the initial position of the contact line, and the dotted lines represent where it was in the previous state. Scale bar is $10 \mathrm{~mm}$.

coefficients in pure water $(S=-2.5 \mathrm{mN} / \mathrm{m})$, and not completely spreading in low-concentration surfactant solution. Therefore, we could produce a macroscopic floating film on an aqueous substrate. Besides, $n$-hexadecane could hardly adsorb near-ultraviolet light ( $365 \mathrm{~nm}$ in our experiment), which would not generate the thermal effect.

To obtain a light-sensitive surface, a cationic photosensitive azobenzene trimethylammonium bromide surfactant (Azo$\mathrm{TAB}$ ) was dissolved in the aqueous substrate. We prepared 1 $\mathrm{mM}$ AzoTAB aqueous solution, which is below the critical micelle concentration of AzoTAB in water $(\mathrm{CMC}=12.6$ and $14.6 \mathrm{mM}$ for trans- and cis-AzoTAB molecules, respectively ${ }^{56}$ ). The experimental setup consists of a $660 \mathrm{~nm}$ coaxial parallel light source, $0.11 \times$ telecentric lens, and a CCD camera for direct observation, as shown in Figure 1. After an alternative UV/blue light stimulus, we observed the floating oil film shrinking and spreading, respectively.

\section{RESULTS AND DISCUSSION}

3.1. Light-Driven Shrinking and Spreading on AzoTAB Solution. A $10 \mu \mathrm{L}$ droplet is deposited on the surface of $1 \mathrm{mM}$ aqueous AzoTAB solution; then, it rapidly spreads and reaches the equilibrium state with a diameter of $36.65 \mathrm{~mm}$ ( $9.5 \mu \mathrm{m}$ calculated thickness) (Figure 2a). After the first $120 \mathrm{~s}$ UV irradiation, the contact line of the film slowly retreats inward at an average speed of $0.025 \mathrm{~mm} / \mathrm{s}$, and a smaller film with a diameter of $33.385 \mathrm{~mm}$ forms (Figure $2 \mathrm{~b}$ ). Next, after the $180 \mathrm{~s}$ exposure to blue light, the oil film spreads slowly at an average speed of $0.017 \mathrm{~mm} / \mathrm{s}$ and forms a bigger film with a diameter of $36.75 \mathrm{~mm}$ (Figure 2c). In these two processes, the maximum of the relative change in diameter $\Delta D / D$ is just about $8.9 \%$ for UV and $9.3 \%$ for blue light. Here, in spite of the small relative change rate of the diameter, we realize the tunable spreading and shrinking process by light stimulus. However, the relative change in diameter $\Delta D / D$ is always less than $10 \%$. It is a crucial problem as to how to get a larger relative change in diameter.

In our experiment, wetting transition occurs during the spreading process. When the droplet touches the solution surface, the initial spreading coefficient is positive $(S=+8.7$ $\mathrm{mN} / \mathrm{m}$ ), and the droplet starts to spread as an oil film. At a later point, we observed that the spreading process stopped, and this indicates that $S$ turns to a negative value $(S<0)$ based on the definition of $S$. Between the two states, there is a transition from $S>0$ to $S<0$, which is caused by the change of air-solution surface tension with its area compressed. We added $10 \mu \mathrm{L}$ of droplets to four different diameter containers containing $1 \mathrm{mM}$ AzoTAB solution (see Supplementary experiment in the Supporting Information). We found that the larger the diameter of the container, the larger the spreading area. This result shows that wetting transition is attributed to the compressed air-solution surface area by droplet spreading. Moreover, it also suggests that there may be an invisible thin film around the finite oil film, which would affect the wettability.

Here, the $n$-hexadecane droplet forms a finite oil film on the substrate, and the initial spreading coefficient $S$ is positive $(S=$ $+8.7 \mathrm{mN} / \mathrm{m}$ ). In relevant reference, it is called pseudopartial wetting configuration, which is an equilibrium state composed of a thin oil film coexisting with the lens of the oil (shown in Figure 3a).The air-water surface is replaced by the oil-water a

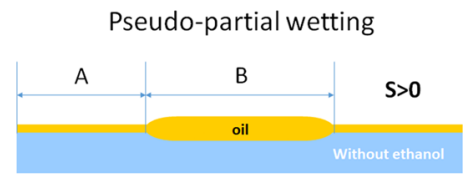

b

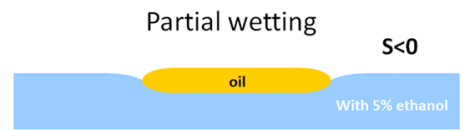

Figure 3. Configuration of pseudopartial wetting without ethanol and partial wetting with $5 \%$ ethanol.

and air-oil interface. In other words, oil-water interfaces and their light response in this regime including $\mathrm{A}$ and $\mathrm{B}$ in Figure $3 \mathrm{a}$ are similar under uniform light irradiation. Therefore, there are almost no resultant forces or interface tension gradient in this case. As a result, photoresponsive wettability is difficult to realize in this case. In our experiment, we can achieve only $10 \%$ relative change in diameter at most.

To realize a remarkable light-controllable wettability, we add ethanol into the substrate. We observe a smaller size of oil film with $S=-2.8 \mathrm{mN} / \mathrm{m}<0$, which is defined as the partial wetting regime (shown in Figure $3 \mathrm{~b}$ ). The effect of ethanol is to decrease the polarity of the substrate and spreading coefficient $S(S<0)$. In this regime, absence of excess oil around the droplet, three interfaces coexist including airwater, air-oil, and oil-water interfaces. The difference in light response properties between oil-water and air-water interface induces the interface tension gradient to drive the lightresponsive wettability.

Through our experiments, $5 \%$ in volume is an appropriate concentration, which could observe a remarkable light-driven 
wetting/dewetting process. More details about the selection of ethanol concentration are analyzed in the Support Information.

3.2. Enhanced Light-Driven Shrinking and Spreading on AzoTAB Solution. We add $5 \%$ ethanol into $1 \mathrm{mM}$ AzoTAB solution and enhance the relative change of diameter $\Delta D / D$. The four pictures in Figure $4 a-d$ give the four
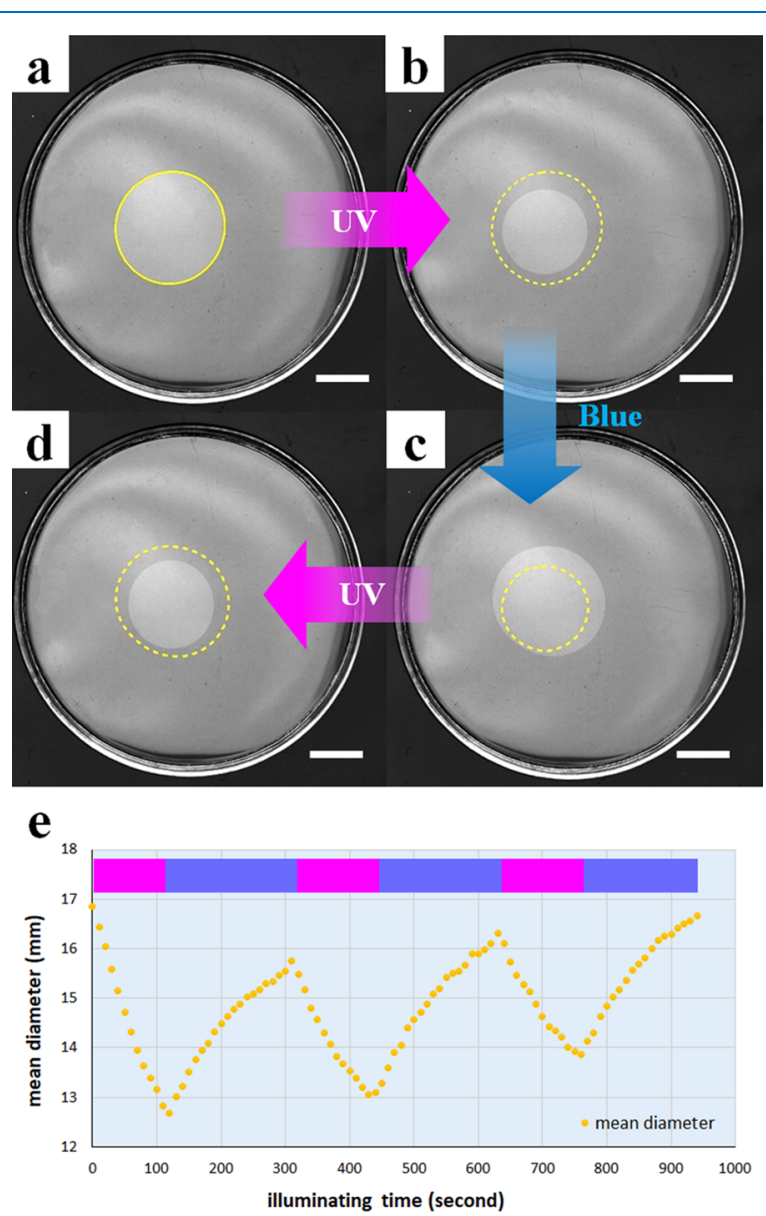

Figure 4. Series of light-induced spreading and shrinking processes of $10 \mu \mathrm{L}$ of $n$-hexadecane droplet on $20 \mathrm{~mL} 1 \mathrm{mM}$ photosensitive surfactant solution are shown in (a-d). After $120 \mathrm{~s}$ UV irradiation, the film shrinks from (a) to (b). Then, the film spreads after $180 \mathrm{~s}$ blue light irradiation and its state turns to (c). The evolution of the film diameter with the illuminating time under alternative $120 \mathrm{~s}$ UV and $180 \mathrm{~s}$ blue light is shown in (e). The scale bar is $10 \mathrm{~mm}$ (the solid line and dashed lines represent the initial position and the position of the previous state, respectively).

successive states of the oil film after the light irradiation. The initial state is shown in Figure 4a, and the spreading area of the droplet is smaller than that without ethanol, as shown in Figure 2a. Same as the experiment above, the film shrinks under UV light (Figure 4a,b) and spreads under blue light (Figure 4b,c) (see the Supplementary video in the Supporting Information).

Figure $4 \mathrm{e}$ gives three cycles of the shrinking and spreading processes. In general, the addition of ethanol makes the oil film smaller but makes the relative change in diameter $\Delta D / D$ greater. The relative change in diameter $\Delta D / D$ exceeds $20 \%$, obviously larger than the $<10 \%$ in the ethanol-free case. In Figure $4 \mathrm{e}$, after the first exposure to UV light, the diameter of the oil film was reduced from 17.28 to $12.8 \mathrm{~mm}$, and the change was about $25.9 \%$ of the initial diameter in $120 \mathrm{~s}$ with its contact line moving uniformly at an average speed of 0.036 $\mathrm{mm} / \mathrm{s}$. Next, when irradiated with blue light, the diameter of the oil film increased from 12.8 to $15.8 \mathrm{~mm}$. In this process, the speed of the contact line gradually and slowly decreased, returning to the starting position. Next, another two same cycles were completed under alternative UV and blue light irradiation. According to the series of experiments, we effectively improved the relative change in diameter $(\Delta D / D$ $>20 \%$ ) by adding ethanol into the substrate, compared with $\Delta D / D<10 \%$ without ethanol. Besides, it takes longer time to achieve the same size of diameter change in the case of blue light (about $180 \mathrm{~s}$ ) than it does for UV (about $120 \mathrm{~s}$ ), and the velocity of the spreading is more slow in blue light. Next, we are going to study the mechanism of light-driven spreading/ shrinking and try to analyze the reason for different moving speeds of contact line under UV or blue light.

3.3. Dynamic Surface and Interface Tension. First of all, we make an analysis of the equilibrium states before and after light irradiation according to the principle of energy, but we do not focus on the intermediate dynamics process during light irradiation because no matter what dynamics process it goes through, the principle of energy is satisfied and the derived results are always valid. So, we do not have to analyze the intermediate process.

Based on the energy principle, the functional relationship between droplet diameter $D$ and spreading coefficient $S$ is obtained (see Supplementary texts in Supporting Information), which is given in eq 1

$$
D=\sqrt[4]{\frac{8 \bar{\rho} g V_{0}^{2}}{-\pi^{2} S}}
$$

We derive geometry parameters of the liquid in the equilibrium state, which is independent of viscosity but depends on the spreading coefficient $S$. It suggests that we can tune the spreading coefficient $S$ by light irradiation to control the wetting and dewetting process. In the first step, the solution was irradiated by UV light, and we notice that the film shrinks because of the reduced spreading coefficient $(\Delta S<0)$; but the film grows after blue light irradiation as a result of the spreading coefficient increasing $(\Delta S>0)$. The series of phenomena is attributed to the change of interfacial tension between two phases after light irradiation.

To verify this point of view mentioned above, we measured the air-solution surface tension and $n$-hexadecane-solution interface tension evolution with irradiation time (shown in Figure 5) using optical contact angle measuring and contour analysis systems (Dataphysics, OCA15EC). The pendant drop method was used in this experiment, analyzing the shape of drops and deducing the surface tension by the Young-Laplace equation.

The result in Figure $5 \mathrm{a}$ indicates that $n$-hexadecane-solution interface tension grows from 33.94 to $36.28 \mathrm{mN} / \mathrm{m}$ under $\mathrm{UV}$, while it falls from 36.16 to $34.36 \mathrm{mN} / \mathrm{m}$ under blue light irradiation in $200 \mathrm{~s}$. But, the result in Figure $5 \mathrm{~b}$ suggests that the air-solution surface tension slowly grows under both UV and blue light in $200 \mathrm{~s}$ with $\Delta \sigma_{\mathrm{w}}<1 \mathrm{mN} / \mathrm{m}$. Considering the definition of spreading coefficient $S=\sigma_{\mathrm{w}}-\left(\sigma_{\mathrm{o}}+\sigma_{\mathrm{ow}}\right)$, we calculate the spreading coefficient $S$ in Figure $5 \mathrm{c}$ according to the data in Figure 5a,b. (Here, air-hexadecane surface tension $\sigma_{\mathrm{o}}$ is constant, equal to $26.8 \mathrm{mN} / \mathrm{m}$, which we measure experimentally). The spreading coefficient falls under UV with the shrinking behavior of the film, while it grows under blue light with the film spreading. The evolution of the spreading 
a

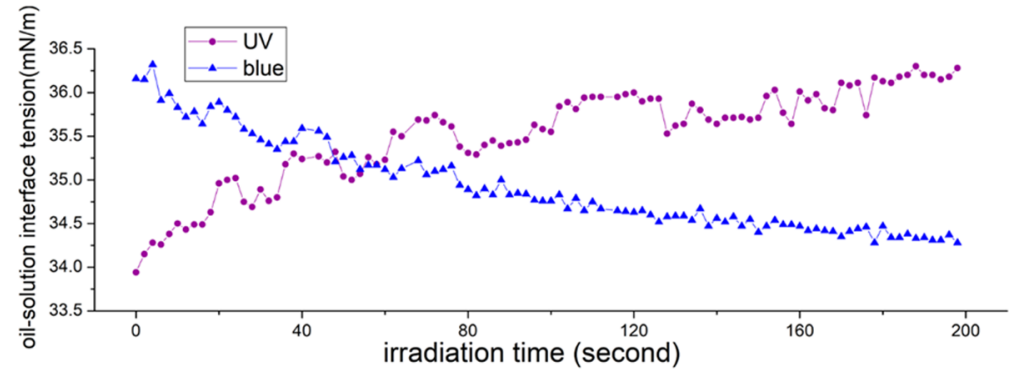

b

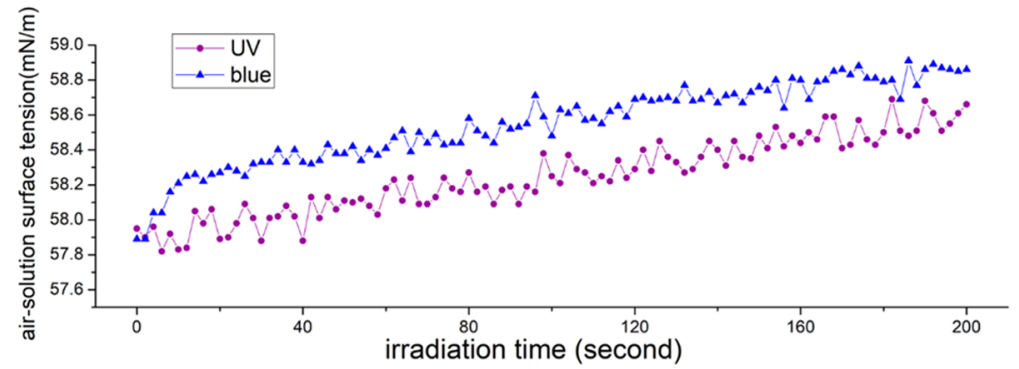

c

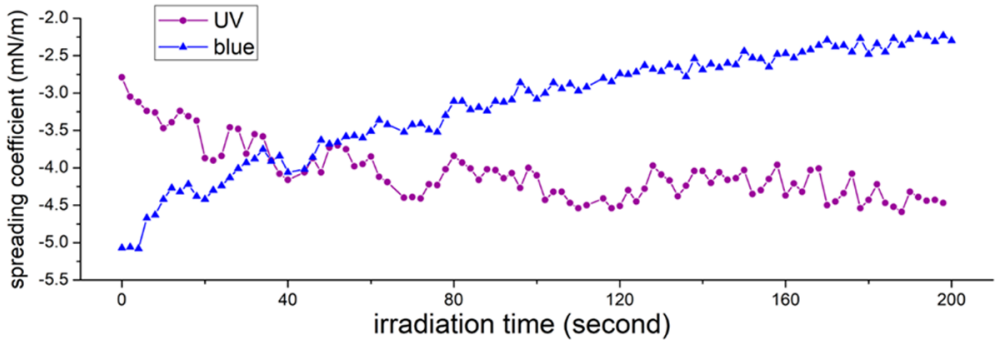

Figure 5. Representative evolution of (a) interface tension between $n$-hexadecane film and AzoTAB substrate solution ( $1 \mathrm{mM}$ AzoTAB solution mixed with 5\% ethanol in volume), (b) surface tension of the $1 \mathrm{mM}$ AzoTAB solution mixed with 5\% ethanol, and (c) spreading coefficient of the system, as functions of time.

coefficient in Figure 5c is consistent with the film wetting behavior.

In other words, the change of the spreading coefficient $(\Delta S$ $\neq 0$ ) means that there are interface tension gradients near the contact line. The difference in light response properties between oil-water and air-water interface induces the interface tension gradient to drive the light-responsive wettability. Under UV light irradiation, oil-water interface tension grows significantly, but the changes of air-water and air-oil surface tension are quite small. As a result, the direction of surface tension gradient and contact line moving are radially inward. Contrary to the former, the surface tension gradient and induced contact line moving are radially outward under blue light irradiation. When the light is turned off, the gradient disappears with the liquid keeping a stationary equilibrium state.

3.4. Photoisomerization and Distribution of AzoTAB Molecules on the Interfaces. The interface tension is an interface property, which is affected by the adsorption and desorption of AzoTAB surfactant molecules. When the surface is stimulated by UV light, a part of adsorbed trans isomers convert into cis rapidly. With cis molecules desorbing from the interface quickly, the surface coverage or adsorbance decreases (Figure 6b). As a result, the interfacial tension of oil-water interface increases, ${ }^{57}$ and the liquid shrinks as the spreading coefficient decreases $(\Delta S<0)$. For the situation under blue light, it is the opposite; the oil-water interface adsorbance increases, and, as a result, the liquid spreads with $\Delta S>0$ (Figure 6c). The change of polarity of AzoTAB molecules $\mathbf{a}$

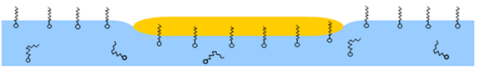

b

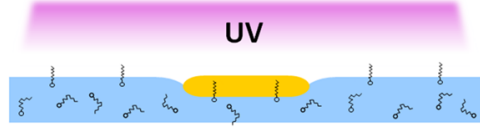

c

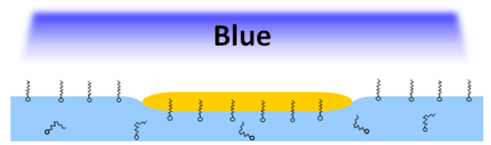

Figure 6. Distribution of surfactants molecules on interface is shown. (a) Initial state without any light irradiation, (b) state after UV light stimulation with cis molecules desorbing from the interface, and the size of oil film becoming smaller, and (c) the state after blue light stimulation with the trans molecules adsorbing to the interface and the size of oil film becoming larger. The photoisomerization of AzoTAB surfactant molecules is shown in the inset.

would lead to different adsorption properties, which is reflected in the change of interface tension and spreading coefficient $S$ in macroscope.

The velocity of spreading or shrinking depends on different adsorption/desorption speeds of two isomers of AzoTAB surfactant molecules. Cis isomers can adsorb and desorb from the interface faster than trans isomers. In relevant references, the cis isomer adsorbs 10 times faster than the trans isomer, but the cis conformation also desorbs 300 times faster. ${ }^{43} \mathrm{UV}$ spectrophotometric measurements showed that when the 
solutions are prepared under UV light $(365 \mathrm{~nm})$ the solution contains $16 \%$ of trans isomers as compared with $66 \%$ of trans isomers under monochromatic blue light (436 nm). Under UV light irradiation, trans isomers convert into cis isomers (the fraction of cis $=84 \%$ in the photostationary state) and desorb from the interface quickly. Under blue light, the molecular configuration is dominated by trans isomers (the fraction of trans $=66 \%$ ), and trans isomers adsorb to the interface more slowly than cis isomers. As a result, the velocity of spreading is slower under blue light illumination in macroscope.

3.5. Spreading Kinetics on Photosensitive Solution. To verify the above suppose about the distribution of surfactants on the surface and identify the differences between the two surfaces irradiated by UV and blue light, we performed another set of experiments. A photosensitive solution substrate (without ethanol) is preilluminated by UV or blue light for 5 min and, then, we deposited $10 \mu \mathrm{L}$ of $n$-hexadecane on it. As soon as the drop is released, the surface of the supporting aqueous solution deforms. From that moment, the droplet starts to spread rapidly and the position of the contact line is recorded. The spreading speed of the contact line is so high that the spreading process is less than $2 \mathrm{~s}$.

Possible power-law spreading dynamics could be revealed by plotting the radius versus time on logarithmic scales, as shown in Figure 7. We observed a spreading process of an acceleration

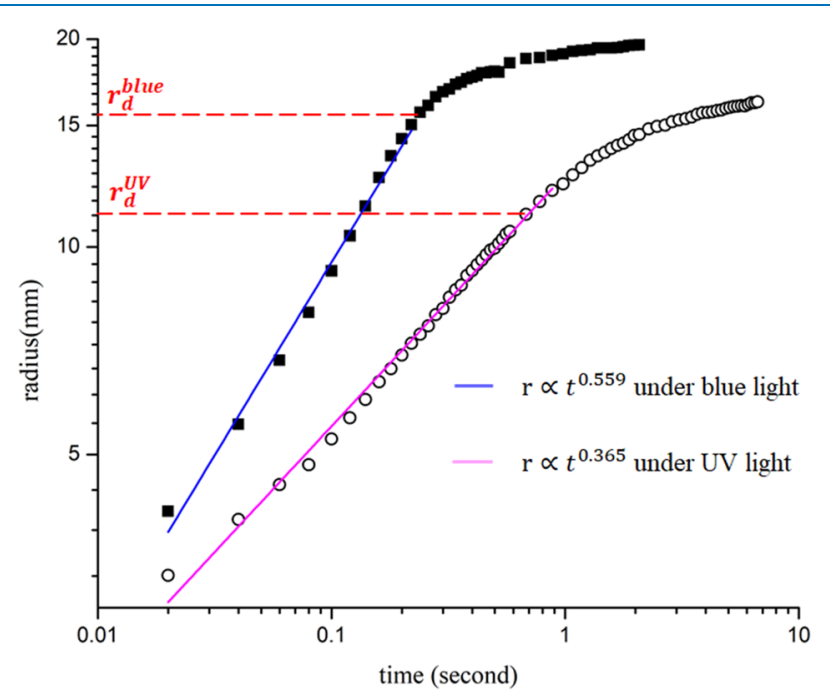

Figure 7. Scaling law of oil droplet spreading on photosensitive solution substrate which is preilluminated by UV or blue light for 5 min. The average scaling exponent for the situation preilluminated by blue light is 0.559 according to linear fitting, which is represented by the blue line $\left(r \propto t^{0.559}\right)$, and 0.365 for the situation preilluminated by $\mathrm{UV}$, which is represented by the purple line $\left(r \propto t^{0.365}\right) . r_{\mathrm{d}}$ is defined as the radius where its slope begins to decay.

followed by deceleration, rather than a clear scaling law with a constant slope. The physical responses of experimental systems are often more complex and, because of the interplay of various forces, only show power-law-like spreading for finite time intervals. To be able to make quantitative comparisons with existing work, we fit this data with straight lines for finite time intervals during the rapid spreading. We find that the average exponents are $0.47<\alpha_{\text {blue }}<0.65$ for blue light regime and 0.27 $<\alpha_{\mathrm{UV}}<0.43$ for UV light regime.

We can contrast this result with power-law spreading of a droplet on a deep viscous support, for which $r(t) \propto t^{3 / 4}$ basing on viscous boundary layer assumption. In this model, the surface tension gradient is balanced by viscous forces, but gravity and inertia can be neglected. ${ }^{58}$ In addition, two other important results are $r(t) \propto t^{1 / 2}$ for the gravity-inertia spreading regime and $r(t) \propto t^{1 / 4}$ for the gravity-viscous spreading regime.

For spreading on blue light preilluminated substrate, $0.47<$ $\alpha_{\text {blue }}<0.65$ indicates that gravity-inertia spreading $(r(t) \propto$ $\left.t^{0.5}\right)$ dominates at the beginning, and then it quickly turns to surface tension-viscous regime $\left(r(t) \propto t^{0.75}\right)$. At the beginning, the spreading coefficient $S$ is positive $(S=+8.7 \mathrm{mN} / \mathrm{m})$, and so the resultant force of interfacial tension in the horizontal direction is outward which drives the droplet spreading. Before $\alpha_{\text {blue }}$ reaches 0.75 , the air-solution surface is compressed for droplet spreading in a finite petri dish. Therefore, there is a possibility that the adsorbance of surfactant molecules per unit area on the surface increases with $S$ decaying quickly during the compression process. As a result, before $\alpha_{\text {blue }}$ reaches 0.75 , the driving force (spreading coefficient) cannot drive the spreading and the contact line brakes with $\alpha_{\text {blue }}$ suddenly decaying to 0.03 . In other words, if the petri dish is large enough (absence of surface compression), $\alpha_{\text {blue }}$ would reach 0.75 , and spreading kinetics would become surface tensionviscous regime.

For spreading on UV light preilluminated substrate, the spreading coefficient $S$ is much smaller than that under blue light $(S=+3.0 \mathrm{mN} / \mathrm{m})$, which could hardly drive the spreading. Therefore, only gravity-driving regime exists such as gravity-inertial or gravity-viscous regime. In our experiment, $0.27<\alpha_{\mathrm{UV}}<0.43$ indicates that gravity, inertia, or viscous force dominates at the same time.

In addition, we find some differences between the two regimes in Figure 7: (1) faster spreading rate and larger equilibrium radius of the droplet for blue light regime, which indicates a better wettability and a larger spreading coefficient $S$ compared with UV regime; (2) a sudden and remarkable decline of the scaling rate for blue light regime, but a relatively gentle process for UV regime; (3) $r_{\mathrm{d}}^{\text {blue }}>r_{\mathrm{d}}^{\mathrm{UV}}\left(r_{\mathrm{d}}\right.$ is defined as the radius where its slope begins to decay) indicates $S_{\text {blue }}>$ $S_{\mathrm{UV}}$, where $S_{\text {blue }}$ and $S_{\mathrm{UV}}$ are the spreading coefficients for blue and UV light regime, respectively. It is remarkable that the sudden brake of the radius for blue light regime might be attributed to the higher surface pressure around the droplet. With the droplet spreading, the air-water surface is compressed rapidly, which induces higher surface pressure and surfactant adsorbance per unit area. The sudden decline of scaling rate for blue light regime also indicates that the adsorbance of surfactant molecules (trans isomers) on the airwater surface is more than that for UV regime, which is consistent with the previous research. ${ }^{43}$ Therefore, there is a relatively gentle deceleration process for UV regime due to its low surface coverage of trans isomers.

\section{CONCLUSIONS}

To summarize, we have observed tunable spreading and shrinking of an oil droplet on a photocontrolled liquid substrate. Besides, we effectively improve the relative change in diameter $(\Delta D / D>20 \%)$ by adding ethanol into the substrate, compared with $\Delta D / D<10 \%$ without ethanol. This photocontrolled spreading and shrinking are induced by the tunable spreading coefficient $S$, which decreases under UV but increases under blue light. Power-law spreading dynamics verifies that $n$-hexadecane has better wettability on the surface 
with high coverage of trans surfactant molecules (irradiated by blue light), while it is poor for the air-solution surface with low coverage of trans surfactants (irradiated by UV light), due to different fraction of conformers and the distribution of surfactant molecules on the interface. The series of experiments provides a basis for a chromocapillary-based optical zoom liquid lens. However, there are still unclear and unsolved problems which may cause difficulties in application. Long response time of the droplet (about $120 \mathrm{~s}$ with the relative change in diameter of $20 \%$ in our experiment) and the spreading/shrinking rate cannot be controlled very well. After many times of alternative spreading and shrinking, the shape of the floating film easily becomes irregular and asymmetric. How to shorten the response time and control accuracy is a crucial topic in the future research of a chromocapillary-based optical zoom liquid lens.

\section{ASSOCIATED CONTENT}

\section{S Supporting Information}

The Supporting Information is available free of charge at https://pubs.acs.org/doi/10.1021/acsomega.9b03039.

\section{An experimental video (MP4)}

Experimental materials, setup, and surface tension measurement; supplementary experiment about the effect of the container size on wetting area; texts about the shape of floating droplets and selection of ethanol concentration (PDF)

\section{AUTHOR INFORMATION}

\section{Corresponding Author}

*E-mail: landing@imech.ac.cn.

\section{ORCID}

Wenjie Ji: 0000-0002-6560-0731

Weibin Li: 0000-0002-6406-1553

Ding Lan: 0000-0001-9688-7731

Notes

The authors declare no competing financial interest.

\section{ACKNOWLEDGMENTS}

We thank Professor Quanzi Yuan (Institute of Mechanics, Chinese Academy of Sciences) for helpful discussions on the theory of wetting of liquid film and reading the manuscript. We gratefully acknowledge financial support from the National Natural Science Foundation of China (Grant Nos. 11472275, 11902321, and U1738118).

\section{REFERENCES}

(1) Harkins, W. D.; Feldman, A. Films the spreading of liquids and the spreading coefficient. J. Am. Chem. Soc. 1922, 44, 2665-2685.

(2) de Gennes, P.-G.; Brochard-Wyart, F.; Quere, D., Capillarity and Wetting Phenomena, 1st ed.; Springer-Verlag; New York, 2004.

(3) Bain, C. D.; Whitesides, G. M. Formation of Monolayers by the Coadsorption of Thiols on Gold - Variation in the Length of the Alkyl Chain. J. Am. Chem. Soc. 1989, 111, 7164-7175.

(4) Daniel, S.; Chaudhury, M. K.; Chen, J. C. Fast drop movements resulting from the phase change on a gradient surface. Science 2001, 291, 633-6.

(5) Ito, Y.; Heydari, M.; Hashimoto, A.; Konno, T.; Hirasawa, A.; Hori, S.; Kurita, K.; Nakajima, A. The movement of a water droplet on a gradient surface prepared by photodegradation. Langmuir 2007, $23,1845-1850$.

(6) Gao, X. F.; Jiang, L. Water-repellent legs of water striders. Nature 2004, 432, 36.
(7) Sun, M.; Chen, Y.; Zheng, Y.; Zhen, M.; Shu, C.; Dai, Z.; Liang, A.; Gorb, S. N. Wettability gradient on the elytra in the aquatic beetle Cybister chinensis and its role in angular position of the beetle at water-air interface. Acta Biomater. 2017, 51, 408-417.

(8) Xia, F.; Jiang, L. Bio-Inspired, Smart, Multiscale Interfacial Materials. Adv. Mater. 2008, 20, 2842-2858.

(9) Sun, M. H.; Luo, C. X.; Xu, L. P.; Ji, H.; Qi, O. Y.; Yu, D. P.; Chen, Y. Artificial lotus leaf by nanocasting. Langmuir 2005, 21, 8978-8981

(10) Han, J. T.; Lee, D. H.; Ryu, C. Y.; Cho, K. W. Fabrication of superhydrophobic surface from a supramolecular organosilane with quadruple hydrogen bonding. J. Am. Chem. Soc. 2004, 126, 47964797.

(11) Shi, F.; Wang, Z. Q.; Zhang, X. Combining a layer-by-layer assembling technique with electrochemical deposition of gold aggregates to mimic the legs of water striders. Adv. Mater. 2005, 17, 1005-1009.

(12) Jiang, L.; Zhao, Y.; Zhai, J. A lotus-leaf-like superhydrophobic surface: A porous microsphere/nanofiber composite film prepared by electrohydrodynamics. Angew. Chem., Int. Ed. 2004, 43, 4338-4341.

(13) Erbil, H. Y.; Demirel, A. L.; Avci, Y.; Mert, O. Transformation of a simple plastic into a superhydrophobic surface. Science 2003, 299, $1377-1380$

(14) Lau, K. K. S.; Bico, J.; Teo, K. B. K.; Chhowalla, M.; Amaratunga, G. A. J.; Milne, W. I.; McKinley, G. H.; Gleason, K. K. Superhydrophobic carbon nanotube forests. Nano Lett. 2003, 3, $1701-1705$

(15) Genzer, J.; Efimenko, K. Creating long-lived superhydrophobic polymer surfaces through mechanically assembled monolayers. Science 2000, 290, 2130-2133.

(16) Nakata, S.; Iguchi, Y.; Ose, S.; Kuboyama, M.; Ishii, T.; Yoshikawa, K. Self-rotation of a camphor scraping on water: New insight into the old problem. Langmuir 1997, 13, 4454-4458.

(17) Bennett, D. E.; Gallardo, B. S.; Abbott, N. L. Dispensing surfactants from electrodes: Marangoni phenomenon at the surface of aqueous solutions of (11-ferrocenylundecyl)trimethylammonium bromide. J. Am. Chem. Soc. 1996, 118, 6499-6505.

(18) Gallardo, B. S.; Gupta, V. K.; Eagerton, F. D.; Jong, L. I.; Craig, V. S.; Shah, R. R.; Abbott, N. L. ELectrochemical principles for active control of liquids on submillimeter scales. Science 1999, 283, 57-60.

(19) Hanczyc, M. M.; Toyota, T.; Ikegami, T.; Packard, N.; Sugawara, T. Fatty acid chemistry at the oil-water interface: Selfpropelled oil droplets. J. Am. Chem. Soc. 2007, 129, 9386-9391.

(20) Lagzi, I.; Soh, S.; Wesson, P. J.; Browne, K. P.; Grzybowski, B. A. Maze Solving by Chemotactic Droplets. J. Am. Chem. Soc. 2010, $132,1198-1199$.

(21) Brzoska, J. B.; Brochardwyart, F.; Rondelez, F. Motions of Droplets on Hydrophobic Model Surfaces Induced by ThermalGradients. Langmuir 1993, 9, 2220-2224.

(22) Cazabat, A. M.; Heslot, F.; Troian, S. M.; Carles, P. Fingering Instability of Thin Spreading Films Driven by TemperatureGradients. Nature 1990, 346, 824-826.

(23) Garnier, N.; Grigoriev, R. O.; Schatz, M. F. Optical manipulation of microscale fluid flow Phys. Rev. Lett. 2003, 91, 054501. DOI: 10.1103/PhysRevLett.91.054501.

(24) Lamhot, Y.; Barak, A.; Rotschild, C.; Segev, M.; Saraf, M.; Lifshitz, E.; Marmur, A.; El-Ganainy, R.; Christodoulides, D. N., Optical Control of Thermocapillary Effects in Complex Nanofluids. Phys. Rev. Lett. 2009, 103. DOI: 10.1103/PhysRevLett.103.264503.

(25) Okawa, D.; Pastine, S. J.; Zettl, A.; Frechet, J. M. J. Surface Tension Mediated Conversion of Light to Work. J. Am. Chem. Soc. 2009, 131, 5396.

(26) Berge, B. Electrocapillarity and Wetting of Insulator Films by Water. Cr Acad. Sci. Ii 1993, 317, 157-163.

(27) Berge, B.; Peseux, J. Variable focal lens controlled by an external voltage: An application of electrowetting. Eur. Phys. J. E 2000, $3,159-163$.

(28) Kuiper, S.; Hendriks, B. H. W. Variable-focus liquid lens for miniature cameras. Appl. Phys. Lett. 2004, 85, 1128-1130. 
(29) Lee, J.; Moon, H.; Fowler, J.; Schoellhammer, T.; Kim, C. J. Electrowetting and electrowetting-on-dielectric for microscale liquid handling. Sens. Actuators, A 2002, 95, 259-268.

(30) Mugele, F.; Baret, J. C. Electrowetting: From basics to applications. J. Phys.: Condens. Matter 2005, 17, R705-R774.

(31) Whitesides, G. M.; Laibinis, P. E. Wet Chemical Approaches To the Characterization of Organic-Surfaces - Self-Assembled Monolayers, Wetting, and the Physical Organic-Chemistry of the Solid Liquid Interface. Langmuir 1990, 6, 87-96.

(32) Wang, R.; Hashimoto, K.; Fujishima, A.; Chikuni, M.; Kojima, E.; Kitamura, A.; Shimohigoshi, M.; Watanabe, T. Light-induced amphiphilic surfaces. Nature 1997, 388, 431-432.

(33) Gu, Z. Z.; Fujishima, A.; Sato, O. Patterning of a colloidal crystal film on a modified hydrophilic and hydrophobic surface. Angew. Chem., Int. Ed. 2002, 41, 2068-2070.

(34) Tadanaga, K.; Morinaga, J.; Matsuda, A.; Minami, T. Superhydrophobic-superhydrophilic micropatterning on flowerlike alumina coating film by the sol-gel method. Chem. Mater. 2000, 12, $590-592$.

(35) Yong, J. L.; Chen, F.; Yang, Q.; Farooq, U.; Hou, X. Photoinduced switchable underwater superoleophobicity-superoleophilicity on laser modified titanium surfaces. J. Mater. Chem. A 2015, 3, 10703-10709.

(36) Yang, Y.; Zhang, L.; Wang, J.; Wang, X.; Duan, L.; Wang, N.; Xiao, F.; Xie, Y.; Zhao, J. Inorganic Surface Coating with Fast Wetting-Dewetting Transitions for Liquid Manipulations. ACS Appl. Mater. Interfaces 2018, 10, 19182-19188.

(37) Ahmad, N. M.; Lu, X. Y.; Barrett, C. J. Stable photo-reversible surface energy switching with azobenzene polyelectrolyte multilayers. J. Mater. Chem. 2010, 20, 244-247.

(38) Ichimura, K.; Oh, S. K.; Nakagawa, M. Light-driven motion of liquids on a photoresponsive surface. Science 2000, 288, 1624-1626.

(39) Jiang, W. H.; Wang, G. J.; He, Y. N.; Wang, X. G.; An, Y. L.; Song, Y. L.; Jiang, L. Photo-switched wettability on an electrostatic self-assembly azobenzene monolayer. Chem. Commun. 2005, 28, $3550-3552$.

(40) Oh, S. K.; Nakagawa, M.; Ichimura, K. Photocontrol of liquid motion on an azobenzene monolayer. J. Mater. Chem. 2002, 12, $2262-2269$.

(41) Diguet, A.; Guillermic, R. M.; Magome, N.; Saint-Jalmes, A.; Chen, Y.; Yoshikawa, K.; Baigl, D. Photomanipulation of a Droplet by the Chromocapillary Effect. Angew. Chem., Int. Ed. 2009, 48, 92819284.

(42) Santer, S. Remote control of soft nano-objects by light using azobenzene containing surfactants. J. Phys. D: Appl. Phys 2018, 51, No. 013002 .

(43) Chevallier, E.; Mamane, A.; Stone, H. A.; Tribet, C.; Lequeux, F.; Monteux, C. Pumping-out photo-surfactants from an air-water interface using light. Soft Matter 2011, 7, 7866-7874.

(44) Mamane, A.; Chevallier, E.; Olanier, L.; Lequeux, F.; Monteux, C. Optical control of surface forces and instabilities in foam films using photosurfactants. Soft Matter 2017, 13, 1299-1305.

(45) Diguet, A.; Li, H.; Queyriaux, N.; Chen, Y.; Baigl, D. Photoreversible fragmentation of a liquid interface for micro-droplet generation by light actuation. Lab Chip 2011, 11, 2666-2669.

(46) Kavokine, N.; Anyfantakis, M.; Morel, M.; Rudiuk, S.; Bickel, T.; Baigl, D. Light-Driven Transport of a Liquid Marble with and against Surface Flows. Angew. Chem., Int. Ed. 2016, 55, 11183-11187.

(47) Venancio-Marques, A.; Barbaud, F.; Baigl, D. Microfluidic Mixing Triggered by an External LED Illumination. J. Am. Chem. Soc. 2013, 135, 3218-3223.

(48) Estevez-Torres, A.; Crozatier, C.; Diguet, A.; Hara, T.; Saito, H.; Yoshikawa, K.; Baigl, D. Sequence-independent and reversible photocontrol of transcription/expression systems using a photosensitive nucleic acid binder. Proc. Natl. Acad. Sci. U.S.A. 2009, 106, 12219-12223.

(49) Lubbe, A. S.; Szymanski, W.; Feringa, B. L. Recent developments in reversible photoregulation of oligonucleotide structure and function. Chem. Soc. Rev. 2017, 46, 1052-1079.
(50) Anyfantakis, M.; Baigl, D. Dynamic Photocontrol of the CoffeeRing Effect with Optically Tunable Particle Stickiness. Angew. Chem., Int. Ed. 2014, 53, 14077-14081.

(51) Chevallier, E.; Monteux, C.; Lequewx, F.; Tribet, C. Photofoams: Remote Control of Foam Destabilization by Exposure to Light Using an Azobenzene Surfactant. Langmuir 2012, 28, 23082312.

(52) Varanakkottu, S. N.; Anyfantakis, M.; Morel, M.; Rudiuk, S.; Baigl, D. Light-Directed Particle Patterning by Evaporative Optical Marangoni Assembly. Nano Lett. 2016, 16, 644-650.

(53) Kopyshev, A.; Lomadze, N.; Feldmann, D.; Genzer, J.; Santer, $\mathrm{S}$. Making polymer brush photosensitive with azobenzene containing surfactants. Polymer 2015, 79, 65-72.

(54) Venancio-Marques, A.; Baigl, D. Digital Optofluidics: LEDGated Transport and Fusion of Microliter-Sized Organic Droplets for Chemical Synthesis. Langmuir 2014, 30, 4207-4212.

(55) Zakrevskyy, Y.; Richter, M.; Zakrevska, S.; Lomadze, N.; von Klitzing, R.; Santer, S. Light-Controlled Reversible Manipulation of Microgel Particle Size Using Azobenzene-Containing Surfactant. Adv. Funct. Mater. 2012, 22, 5000-5009.

(56) Diguet, A.; Mani, N. K.; Geoffroy, M.; Sollogoub, M.; Baigl, D. Photosensitive Surfactants with Various Hydrophobic Tail Lengths for the Photocontrol of Genomic DNA Conformation with Improved Efficiency. Chem. - Eur. J. 2010, 16, 11890-11896.

(57) Baigl, D. Photo-actuation of liquids for light-driven microfluidics: state of the art and perspectives. Lab Chip 2012, 12, 36373653.

(58) Berg, S. Marangoni-driven spreading along liquid-liquid interfaces. Phys. Fluids. 2009, 21, No. 032105. 